\title{
O CONSUMO DE ADOÇANTES EM PRODUTOS ALIMENTÍCIOS NA CIDADE DE CURITIBA
}

\section{THE CONSUMPTION OF SWEETENERS IN FOOD PRODUCTS ON CURITIBA CITY}

\author{
CAMPOS, Caroline Carneiro ${ }^{1 *}$, DEGÁSPARI, Cláudia Helena ${ }^{2}$, MOTTIN, Fatima ${ }^{3}$ \\ ${ }^{1}$ Aluna de Graduação do Curso de Nutrição da Universidade Tuiuti do Paraná \\ Rua Sargento Lázaro Rodrigues, 850 - São José dos Pinhais - PR. \\ e-mail: carol_campos_1@hotmail.com \\ 2 Professora Adjunta da Universidade Tuiuti do Paraná. \\ e-mail: sensus@cwb.matrix.com.br \\ 3 Professora Substituta da Universidade Federal do Paraná \\ e-mail: mottin_fatima@hotmail.com
}

RECEBIDO: AGO/07 ACEITE: STE/07

\begin{abstract}
RESUMO
Este artigo apresenta uma pesquisa de opinião para verificar os motivos que levam uma pessoa a consumir produtos diet ou adicionados de adoçantes em substituição aos produtos adoçados naturalmente.

A pesquisa se justifica à medida que busca analisar quais os motivos que levam uma pessoa com saúde normal, consumir produtos diet ou com redução de carboidratos, além dos problemas de patologia do tipo diabetes e obesidade, analisando se a população está aderindo o uso de adoçantes em prol da saúde, através de recomendação médica ou de nutricionista, ou se está usando apenas pela estética e por idéia própria, tendo consciência das vantagens e desvantagens de seu uso. Para a pesquisa, foi aplicado um instrumento de avaliação contendo 11 perguntas fechadas sobre: renda familiar, grau de instrução, peso, altura, qual motivo faz uso dos produtos diet, preferências por algum tipo de adoçante e se possuem alguma patologia. A amostra foi constituída de 100 respondentes, selecionados aleatoriamente, entre homens e mulheres. Os resultados indicam que os entrevistados se concentram entre 15 e 44 anos, somando $75 \%$. Destes, $60 \%$ utilizam produtos diet sempre ou às vezes não tendo um padrão quanto ao motivo pelo qual usam se por estética ou pela saúde. Os maiores consumidores são as mulheres, representando $65 \%$ dos entrevistados. Em relação à renda mostrou-se o esperado, que produtos diet são mais consumidos por pessoas que tem renda familiar alta, ou seja, estes produtos ainda são considerados "caros" pela população sendo comprados apenas quando necessário. A maioria dos entrevistados classifica-se pelo índice de massa corpórea na classe de eutrofia, porém $41 \%$ deles apresentam algum tipo de obesidade e apenas $13 \%$ deles sentem-se obesos e citam como uma patologia.

Conclui-se que independente de renda, de instrução, de sexo, idade e presença ou não de patologias, o uso desses produtos, deve ser orientado por médicos e/ou nutricionistas, para que se faça uso correto destes produtos a fim de se obterem resultados satisfatórios.
\end{abstract}

Palavras-chaves: Adoçantes; produtos diet; pesquisa de consumo. 


\begin{abstract}
This article presents an opinion research to study the reasons that influence the decision of a person to consume products diet or added of sweeteners in substitution to the products sweetened naturally.

The research looks to analyze which the reasons that make a person with normal health to consume products diet or with reduction of carbohydrates, besides the problems of pathologies like Diabetes and Obesity. Analyzing if the population is adhering the use of sweeteners on behalf of the health, through medical recommendation or of a nutritionist, or just for the esthetics sake and for own idea, being conscious of the advantages and disadvantages of its use.

For the research a questionnaire with eleven items was applied with questions on: family income, instruction degree, weight, height, reason to use the diet products, preferences for sweetener type and if they have some pathology. The sample was constituted of 100 customers randomly selected, between men and women. The results show that the interviewees are mostly between 15 and 44 years old, adding $75 \%$. Of these, $60 \%$ use products diets always or eventually, without a clear pattern as for the reason for which they are used either for esthetics or for health reasons. The main consumers are the women, representing $65 \%$ of the interviewees. In relation to income, the expected was shown: that diet products diet are more consumed by people with high income, in other words, these products are still considered expensive by the population, being only bought when necessary. Most of the interviewees are classified for the index of corporal mass in the eutrophy class, however $41 \%$ of them present some obesity type and only $13 \%$ of them feel obese and mention one pathology. It is concluded that independent of income, instruction degree, sex, age and presence or absence of pathologies the use of those products should be guided by doctors and/or nutritionists, to make correct use of these products in order to they obtain satisfactory results.

Key words: Sweetener; diet products; research of consumption.
\end{abstract}

\title{
1. INTRODUÇÃO
}

Os produtos diet, light, low, free há algum tempo avolumam-se nas prateleiras dos supermercados, chamando atenção de pessoas preocupadas com a saúde e a estética. O consumidor, entretanto, não está suficientemente esclarecido sobre o significado desses termos e sente-se pouco seguro em utilizar tais alimentos (CÂNDIDO \& CAMPOS, 1995).

De acordo com a legislação brasileira em vigor, o termo dietético possui sentido amplo e não se aplica apenas aos alimentos isentos de açúcar, ou de baixas calorias. Para exemplificar, são considerados dietéticos: alimentos para dietas de restrição de açúcares, gorduras, colesterol, sódio, aminoácidos ou proteínas; alimentos para dietas de controle de peso; e alimentos para dietas enterais (CÂNDIDO \& CAMPOS, 1995).

O termo diet pode, opcionalmente, ser utilizado em alimentos produzidos para indivíduos com exigências físicas e/ou que sofrem de doenças específicas como, por exemplo, diabetes. Nesses casos podem ser incluídos, alimentos indicados para as dietas com restrição dos nutrientes: carboidrato, gordura, proteínas e sódio; alimentos exclusivamente empregados para controle de peso e alimentos para dieta de ingestão controlada de açúcar (INMETRO, 2004).

Consumidores atualmente ainda classificam os dietéticos como alimentos que não contém açúcar, de baixa caloria, destinados a quem faz dieta para manter o peso (CÂNDIDO \& CAMPOS, 1995).

O Brasil também foi invadido pela onda diet, mas só teve um crescimento explosivo em 1988 após modificações na legislação brasileira que passou a considerar dietéticos como alimentos e não mais como medicamentos (CÂNDIDO \& CAMPOS, 1995).

A rotulagem dos alimentos dietéticos deve ser facilmente compreensível e adequada ao consumo a que o produto se propõe, evitando possíveis induções a erros que levem a riscos de saúde pública (CÂNDIDO \& CAMPOS, 1995).

Tendo em vista que produtos diet, que antes eram utilizados como medicamentos, hoje estão sendo usados à vontade como alimento desde que a legislação foi modificada. Objetiva-se neste estudo desenvolver um trabalho de avaliação do nível de consumo de adoçantes em produtos alimentícios, no sentido de melhor conhecer os consumidores, avaliando principalmente o conhecimento destes consumidores sobre o uso dos diferentes adoçantes encontrados no mercado e verificar se estes consumidores o utilizam corretamente, com a finalidade de cuidar da saúde ou da estética (CÂNDIDO \& CAMPOS, 1995).

\section{METODOLOGIA}

Para o desenvolvimento deste trabalho, foi aplicado um instrumento de avaliação com perguntas específicas ao tema (uso de adoçantes) e com uma pesquisa de mercado, para identificar os diferentes tipos de adoçantes encontrados nos produtos alimentícios, embasado por uma análise estatística adequada. 
O instrumento de avaliação possui 11 perguntas objetivas sobre: faixa etária, escolaridade, grau de instrução, consumo de produtos diet, uso de adoçantes e atividade física (vide ANEXO 01).

Foram entrevistadas 100 pessoas, sendo os locais de coleta dos dados academia de ginástica e unidades de saúde. Os dados coletados foram analisados estatisticamente para que se tivesse uma interpretação científica. (TRIOLA, 1999; AMERINE et al.,1965).

Não houve exclusões de pessoas durante a aplicação do mesmo. Todas as pessoas entrevistadas foram incluídas na pesquisa. A abordagem na academia de ginástica e unidades de saúde foi realizada na entrada, de maneira aleatória, podendo a pessoa responder o questionário como uma entrevista ou então ela própria respondendo, de acordo com a preferência. Qualquer pessoa que chegasse ao local era abordada sobre o objetivo da pesquisa e respondia o instrumento de avaliação se realmente estivesse interessada em fazê-lo.

Após o trabalho realizado e concluído, o resultado esperado era que a população estivesse utilizando adoçantes em produtos alimentícios, por recomendação de médico e/ou nutricionista em prol da saúde, devido a patologias tais como: diabetes e obesidade, tendo consciência das vantagens e desvantagens de seu uso.

\subsection{TECNICA ESTATÍSTICA UTILIZADA PARA ANÁLISE}

Para análise da pesquisa foi utilizada estatística descritiva e teste qui-quadrado $\left(X^{2}\right)$. $O$ teste $X^{2}$ aplicável em dados representados em forma de freqüência, é utilizado para detectar significância estatística da diferença entre dois grupos independentes.

Onde: $O=$ Freqüência observada

$$
\begin{aligned}
& \text { Estatística do teste } \Rightarrow \chi^{2}=\frac{\sum(O-E)^{2}}{E} \\
& \text { Observada }
\end{aligned}
$$

$$
E=\text { Freqüência esperada }
$$

O teste $\chi 2$ (teste qui-quadrado) é uma estatística não paramétrica de fácil compreensão e muito usada para verificar se há diferença entre freqüências esperadas e freqüências observadas de duas ou mais categorias. No caso, a freqüência esperada é sempre aquela que determina a igualdade dos grupos. O teste conflita a freqüência esperada da observada, e se forem verificadas diferenças significativas entre as duas categorias, os grupos são considerados diferentes.

\section{DISCUSSÃO DOS RESULTADOS}

Pelo levantamento realizado na pesquisa, verificou-se que $54 \%$ dos entrevistados foram do sexo feminino e $45 \%$ do sexo masculino, conforme demonstrado do GRÁFICO 01.

GRÁFICO 01: SEXO DOS ENTREVISTADOS.

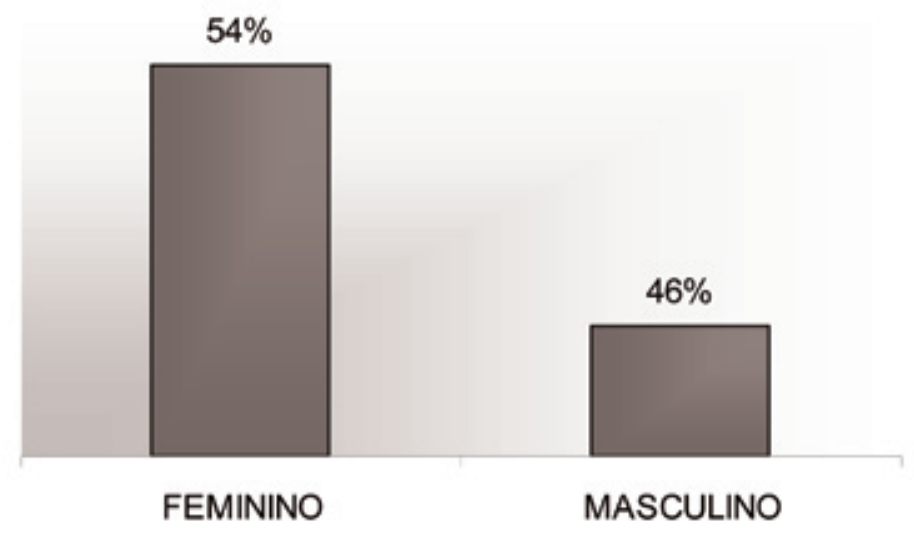


O perfil do público entrevistado apresenta-se no GRÁFICO 02, sendo que abrangeu o público mais jovem, com uma tendência decrescente com o aumento da idade.

GRÁFICO 02: FAIXA ETÁRIA DOS ENTREVISTADOS.

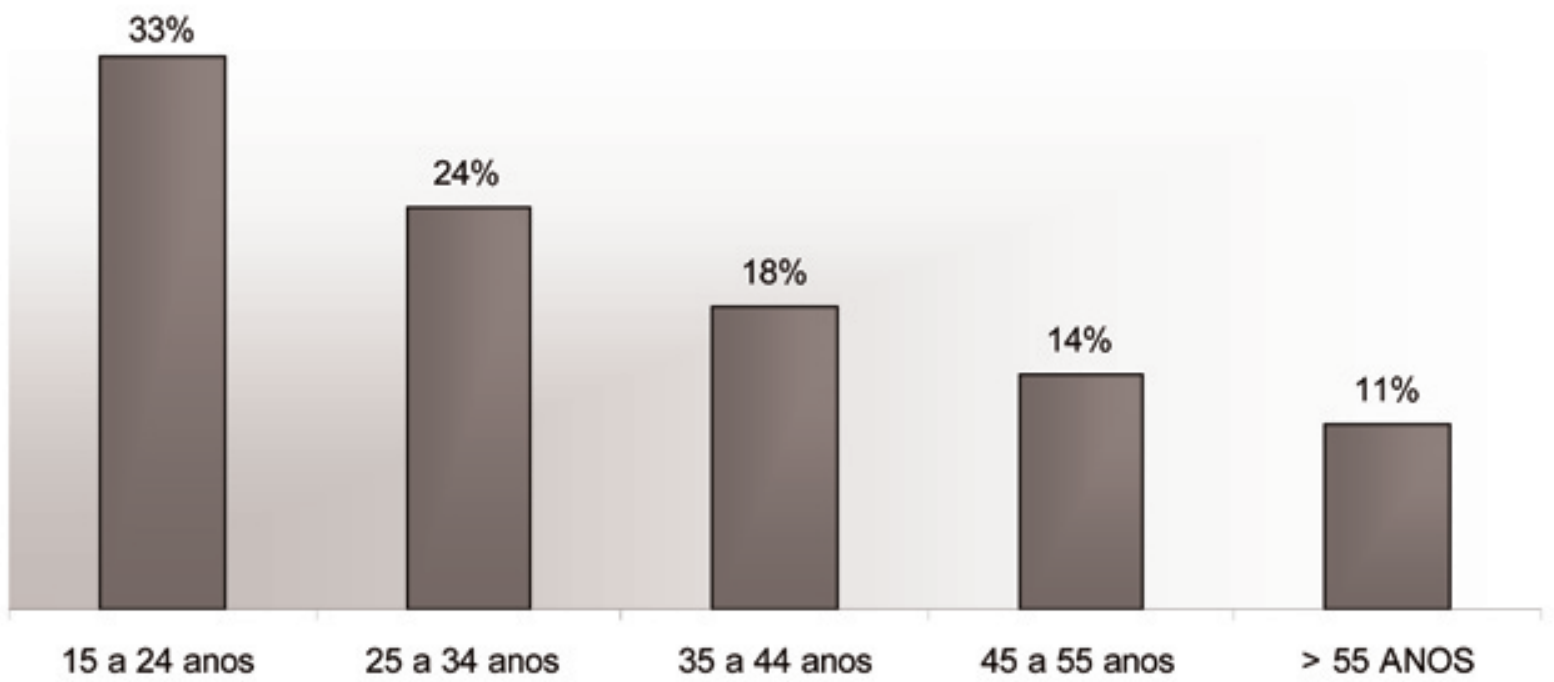

A maioria dos entrevistados apresentou uma classificação em relação ao peso e a altura de eutrófico, e ainda, a amostra mostra um grupo significativo de algum tipo de obesidade, com $41 \%$ dos entrevistados sendo classificados com obesidade II, obesidade I, pré-obesidade, o que denota que a amostra não possui um grupo predominante se comparado aos eutróficos com os de algum tipo de obesidade. Outra observação é que o grupo de magreza é minoritário entre os entrevistados.

GRÁFICO 03: CLASSIFICAÇAO DO ÍNDICE DE MASSA CORPÓREA (IMC) DOS ENTREVISTADOS.

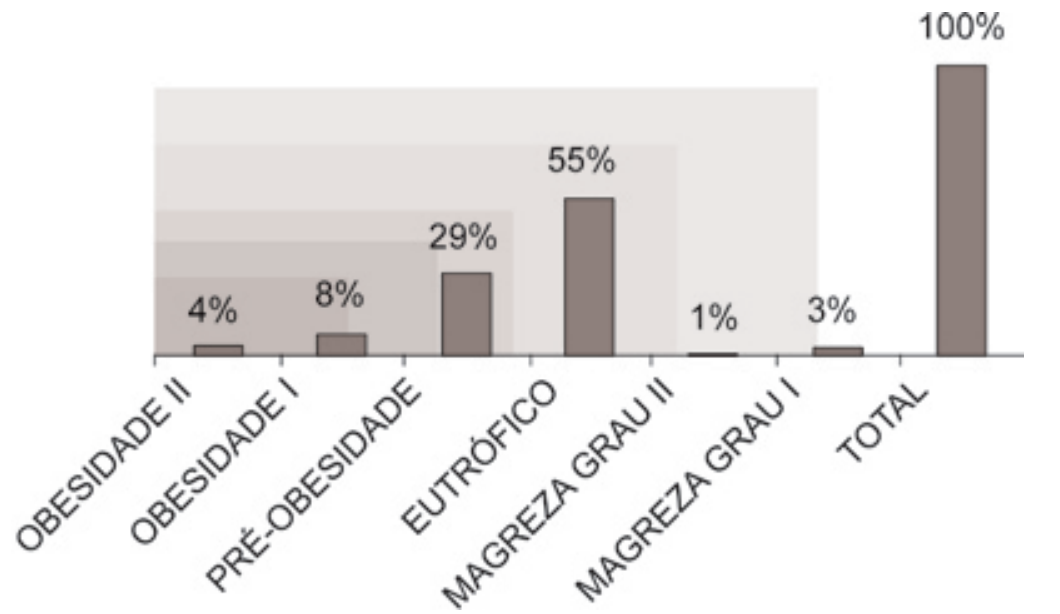

OBS: Os pesos para cálculo de índice de massa corpórea (IMC) foram fornecidos por cada pessoa entrevistada, não sendo realizado uma avaliação antropométrica.

A dispersão da faixa salarial dos entrevistados mostra uma concentração de $46 \%$ deles ganhando entre 2 e 5 salários e outros $24 \%$ ganhando acima de 8 salários mínimos. 


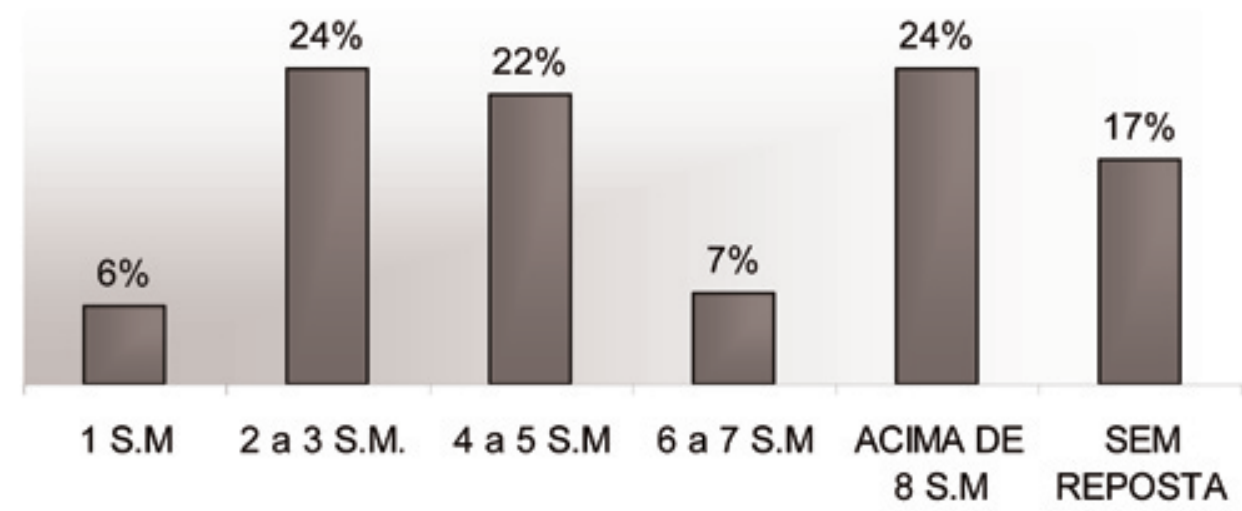

A escolaridade dos entrevistados está concentrada em $3^{\circ}$ grau completo e incompleto. Com o teste $x^{2}$ temse que a freqüência esperada se difere da freqüência observada, o que nos permite concluir que ter iniciado ou terminado o $3^{\circ}$ grau é uma característica predominante nessa amostra, com uma confiança maior que $99 \%$.

GRÁFICO 05: ESCOLARIDADE DOS ENTREVISTADOS.

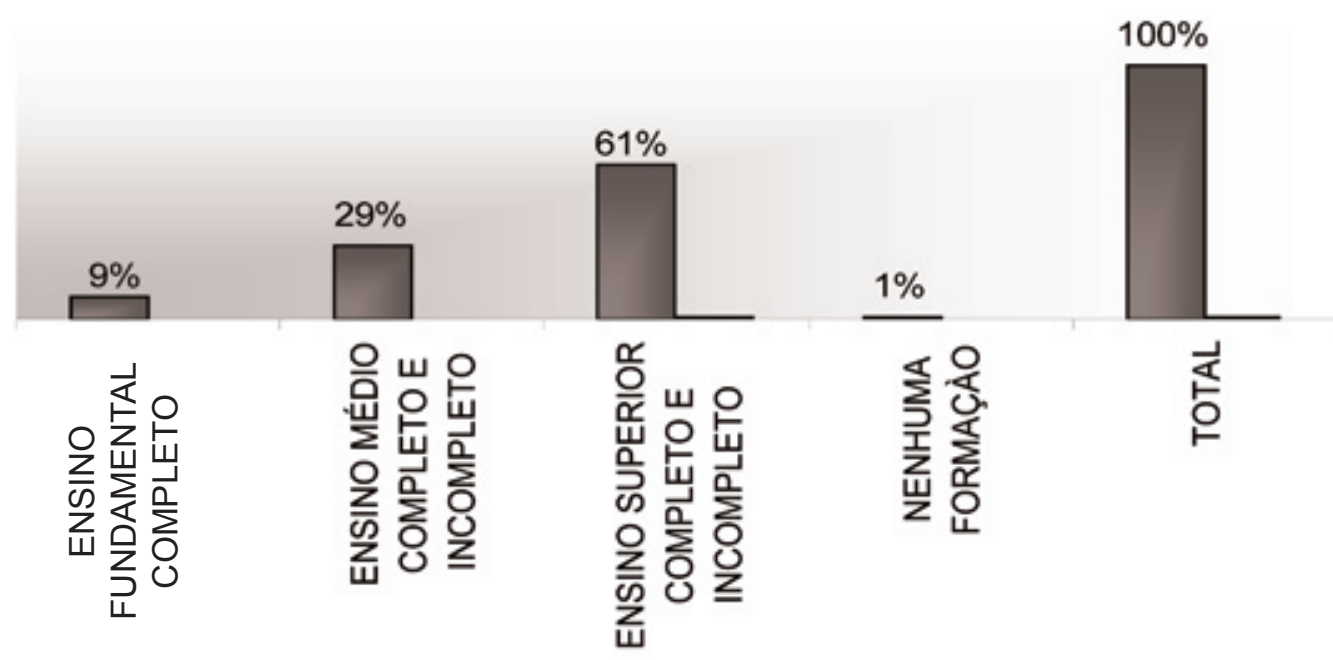

O GRAFICO 06 mostra que a maioria dos entrevistados freqüenta academia de ginástica, isso por que foi um dos locais escolhidos para coletar a amostra. 


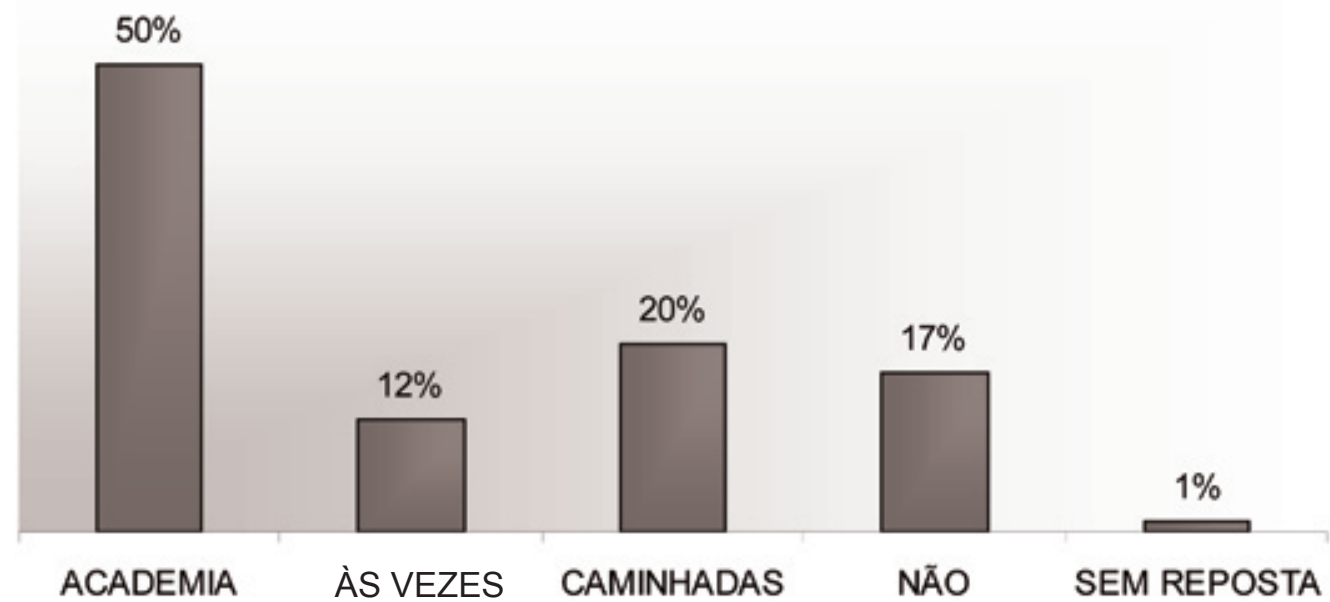

Entre os entrevistados, $60 \%$ afirmam utilizar produtos diet sempre ou às vezes.

GRÁFICO 07: UTILIZAÇÃO DE PRODUTOS DIET PELOS ENTREVISTADOS.

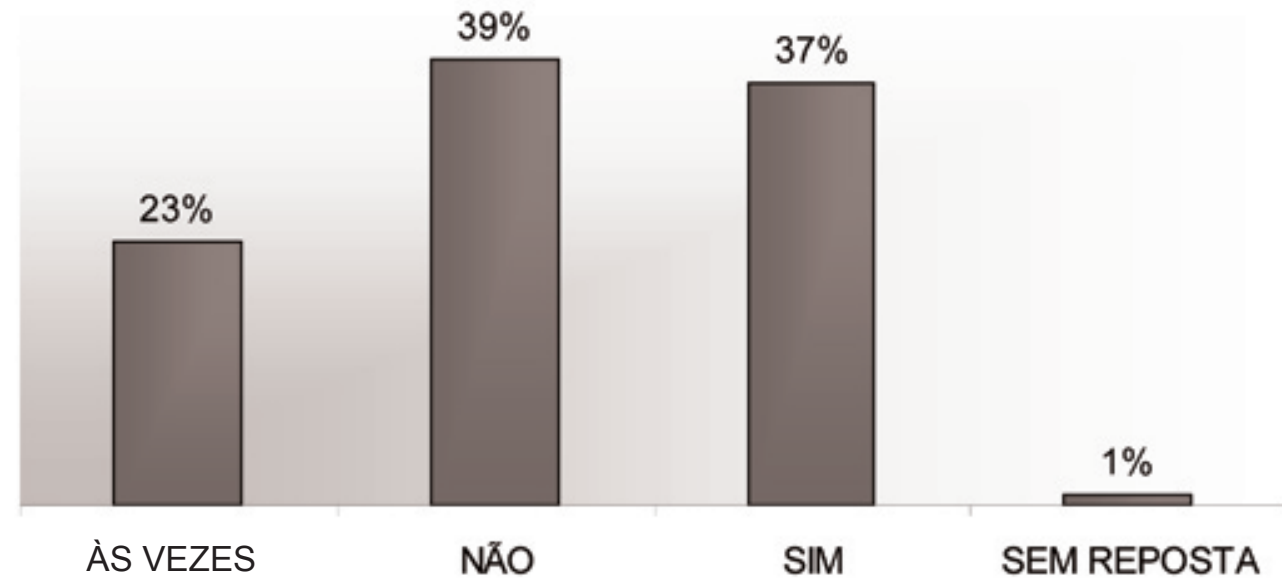

Dos 60 entrevistados que utilizam produtos diet freqüentemente ou às vezes, a maioria utiliza pensando tanto na estética como na saúde. Aplicando o teste $\chi^{2}$ observa-se que não existe um padrão para utilização, podese afirmar que as pessoas utilizam pra ambas as finalidades, isso com uma confiança de mais de $99 \%$. 


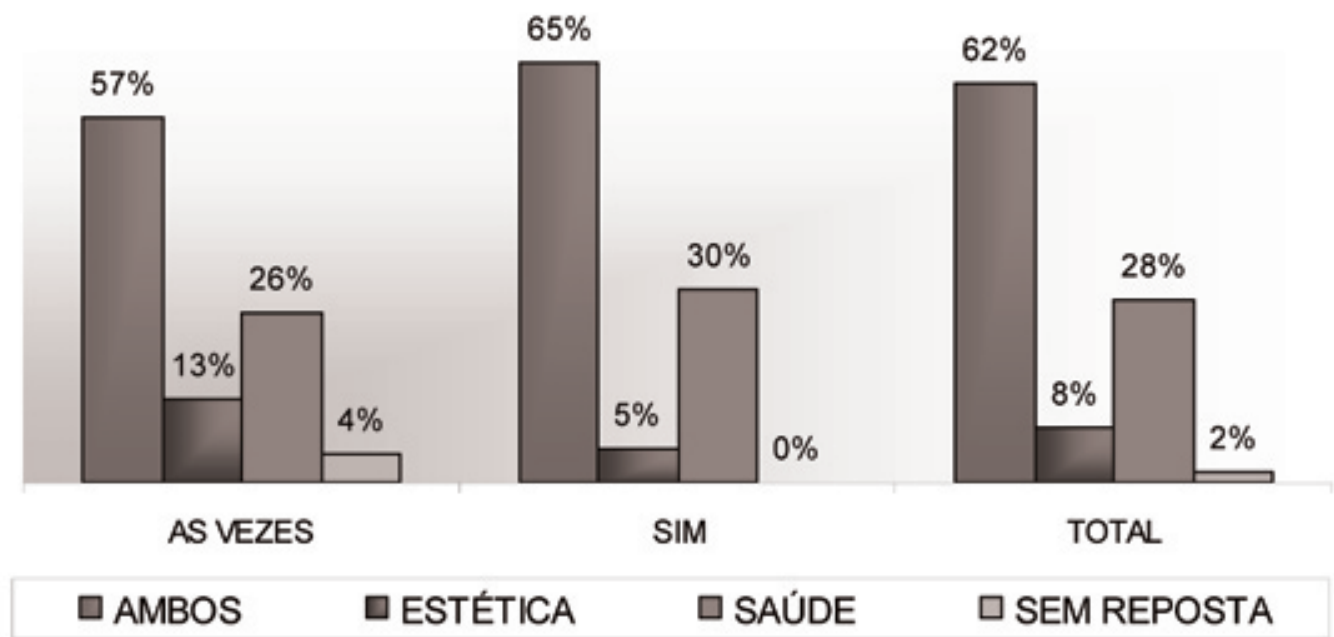

Da mesma forma, dos 60 entrevistados que utilizam produtos diet freqüentemente ou às vezes, a maioria utiliza por conta própria e apenas $31 \%$ utilizam por algum tipo de recomendação médica. Aplicando o teste $X^{2}$ confirma-se que é uma característica típica das pessoas utilizarem os produtos diet sem recomendação médica.

GRÁFICO 09: RECOMENDAÇÃO PARA USO DO PRODUTO PELOS ENTREVISTADOS.

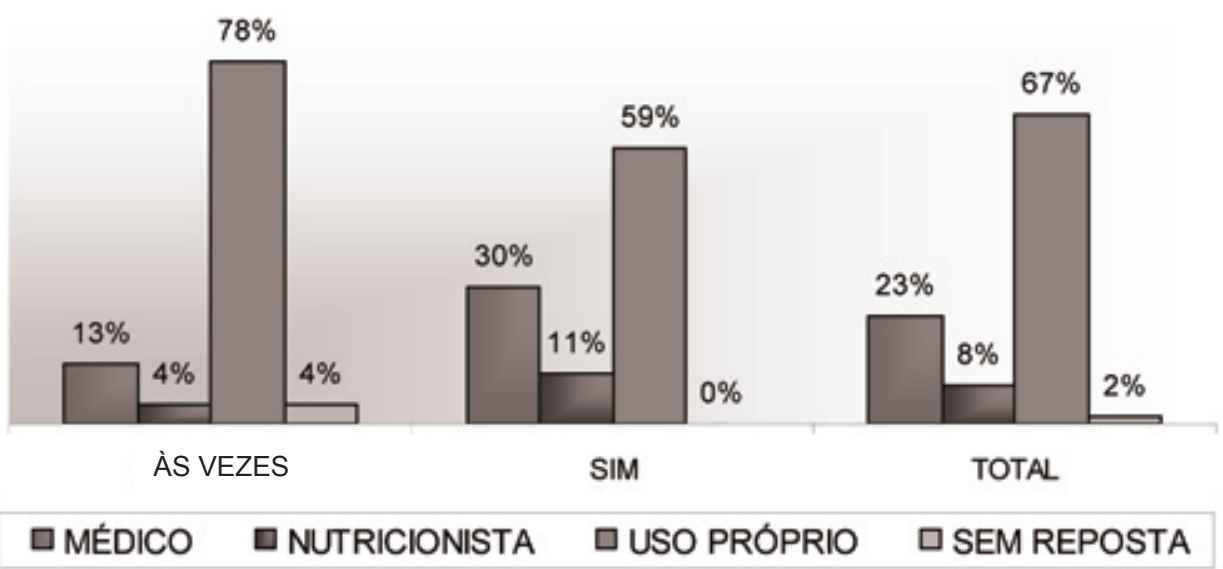

O GRAFICO 10 demonstra que a maioria dos entrevistados não possui patologias. 


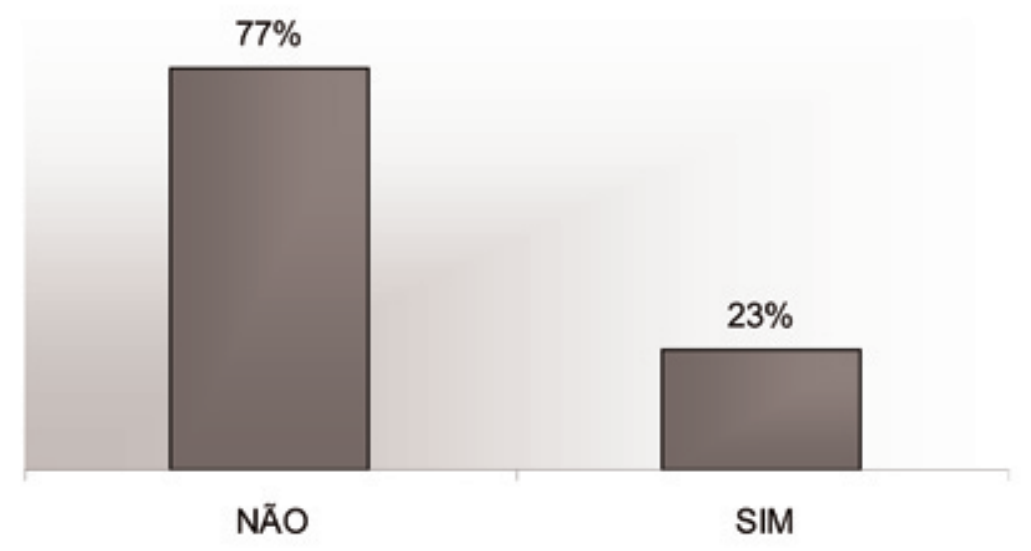

Observando o GRÁFICO 11, do total de 60 entrevistados que utilizam produtos diet, verifica-se que a preferência por algum tipo de adoçante não apresenta tendência, isso comprovado com o teste $X^{2}$. Porém, quando se olha dentro da freqüência de uso, observa-se uma possível tendência, que poderia a vir se confirmar com uma amostra maior. Com essa amostra, nota-se uma tendência, confirmada com uma confiança de $93 \%$, no caso das pessoas que usam sempre (representadas pela resposta sim). $O$ teste $X^{2}$ afirma que essas pessoas têm uma preferência, já no caso das pessoas que utilizam às vezes, a porcentagem mostra a tendência, mas o teste não encontra essa diferença significativa, pois a amostra não se mostrou suficiente.

\section{GRÁFICO 11: TIPO DE PATOLOGIA DOS ENTREVISTADOS.}

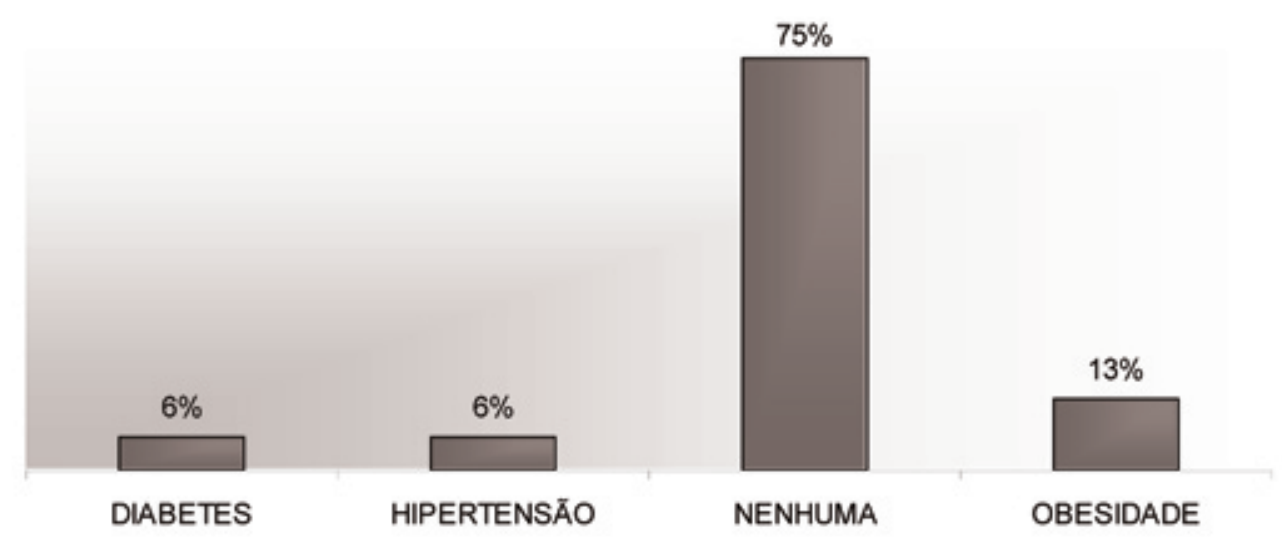

Entre as patologias citadas, a obesidade é a que mais se apresenta nos entrevistados. Um comentário interessante é que se for observado o índice de massa corpórea das pessoas (GRÁFICO 03), elas apresentam algum tipo de obesidade ( $41 \%$ dos entrevistados da amostra). Desses, $46 \%$ não citaram na pergunta "patologia" terem obesidade, o que mostra que pode haver uma dificuldade em identificar o que é uma pessoa obesa ou pré-obesa por parte dos entrevistados. 
GRÁFICO 12: PREFERÊNCIA POR ADOÇANTES.

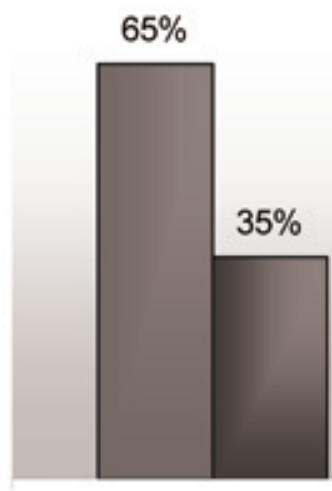

ÀS VEZES

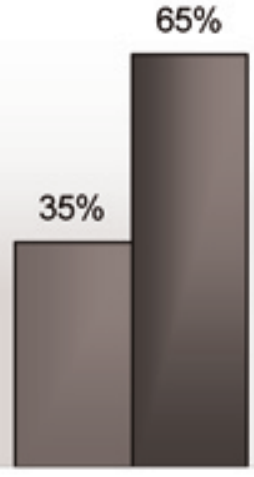

SIM

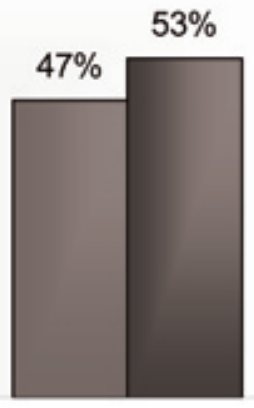

TOTAL

\section{口NÃO घSIM}

Em relação a preferência por alguma marca, a mais citada foi "Zero Cal", porém dos que usam os produtos diet, a maioria $47 \%$ não citou nenhuma preferência.

GRÁFICO 13: MARCA DE ADOÇANTES.

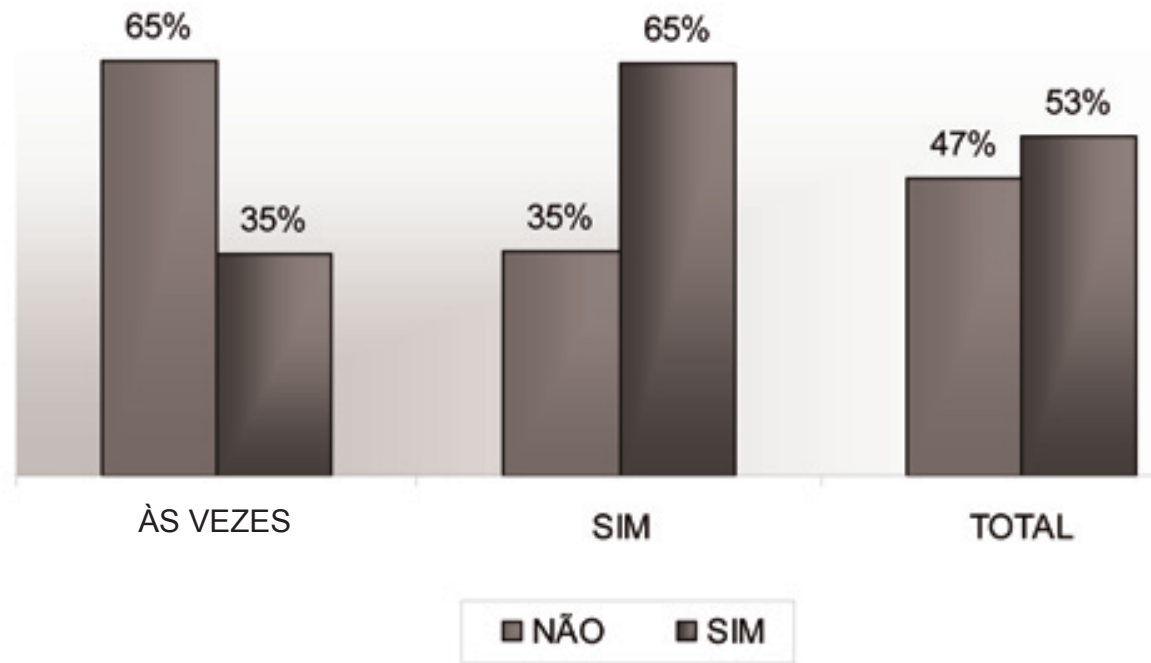

O cruzamento de dados entre o consumo de produtos diet e o fato de apresentar alguma patologia, entre os que utilizam produtos diet, o índice de patologia é mais alto, estando na marca de $35 \%$. 


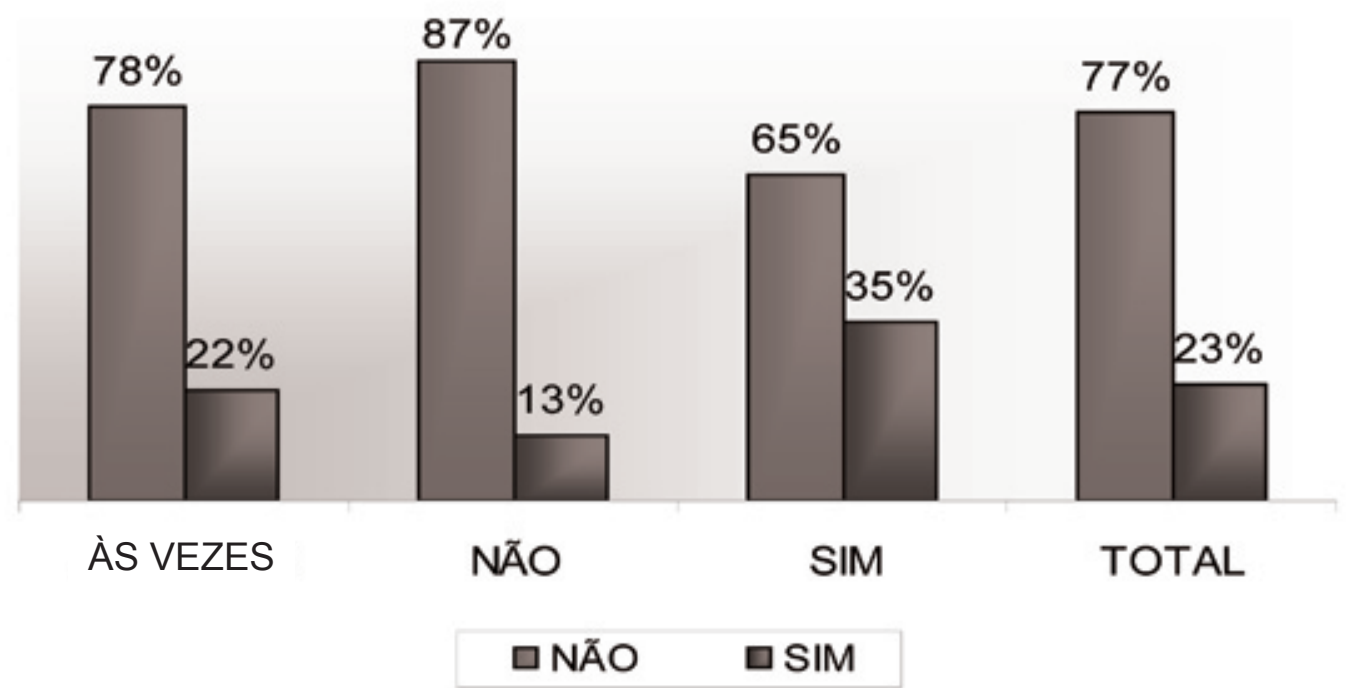

Analisando o cruzamento dos dados tem-se que todos os diabéticos utilizam adoçante, que os hipertensos não costumam usar e os $52 \%$ dos obesos utilizam.

GRÁFICO 15: PATOLOGIA DOS ENTREVISTADOS COM O USO DE PRODUTOS DIET.

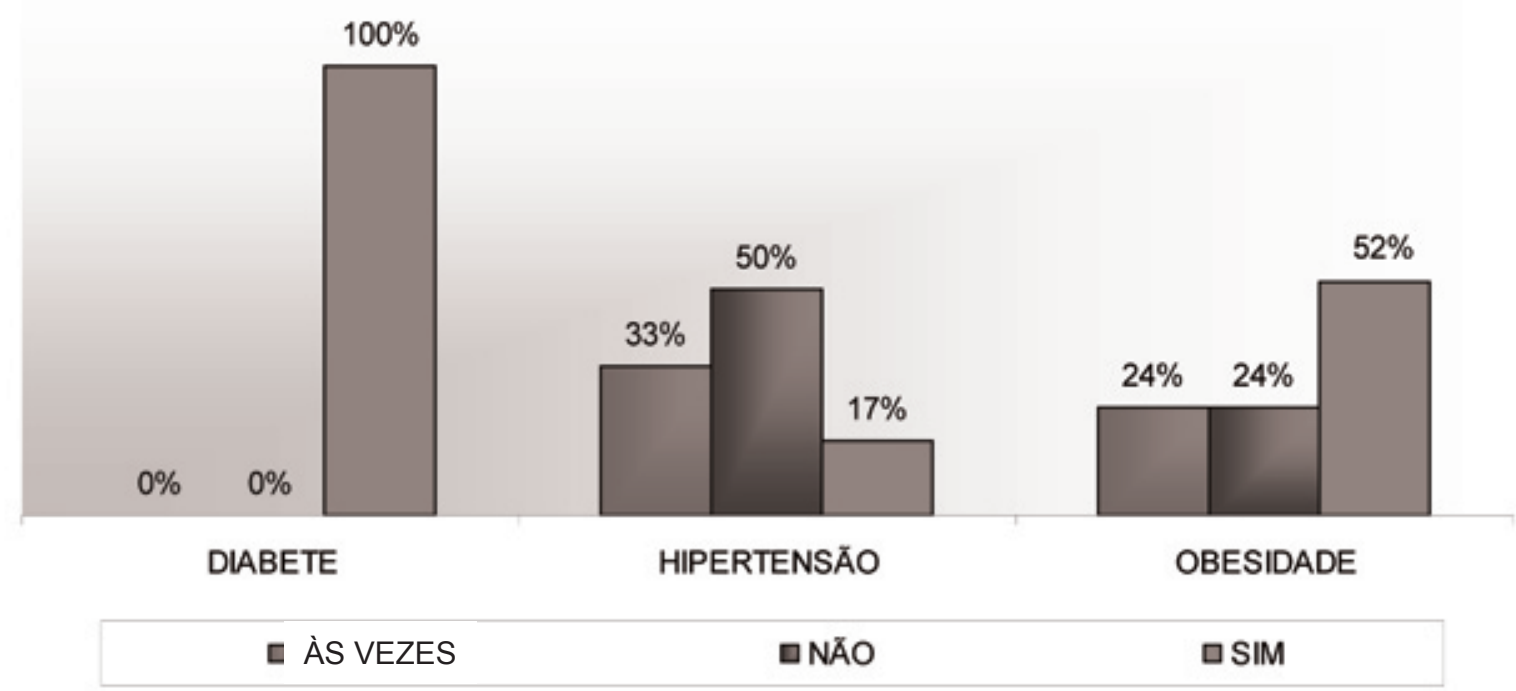

Comparando-se alguma tendência em consumo em relação a sexo, observa-se apenas que as mulheres apresentam um consumo mais freqüente do que os homens, também confirmado com um teste $X^{2}$ com uma confiança de $93 \%$. 
GRÁFICO 16: CONSUMO DE PRODUTOS DIET EM RELAÇÃO AO SEXO DOS ENTREVISTADOS.

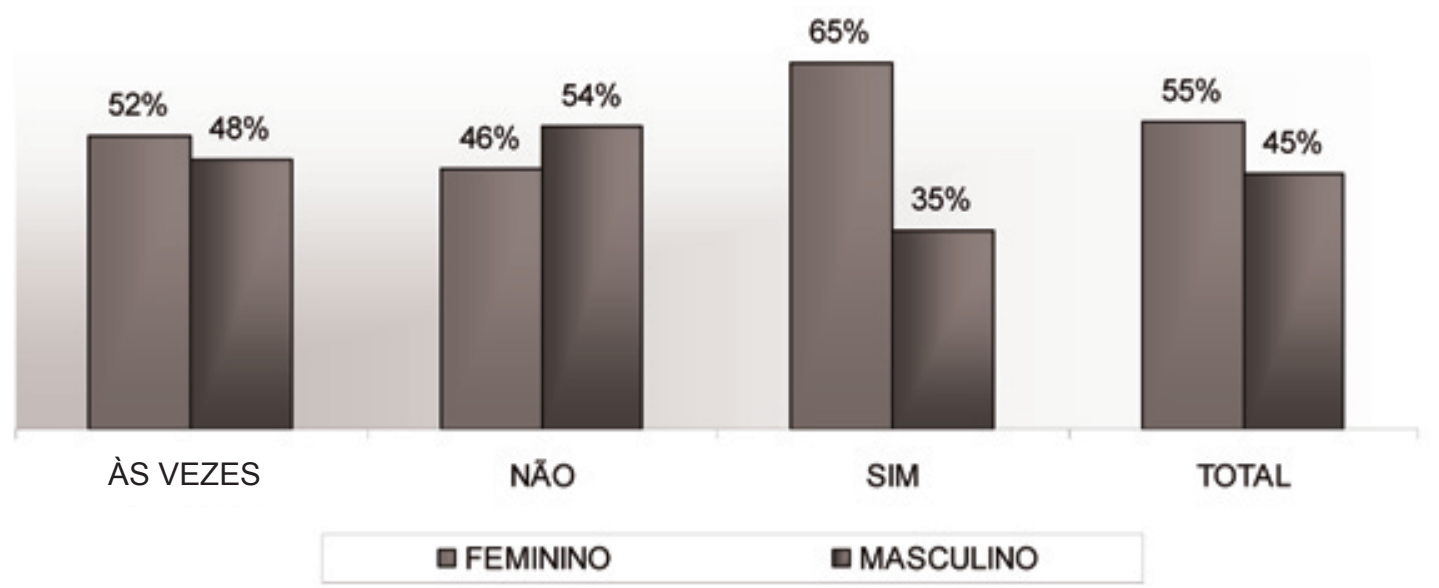

Em relação à faixa etária, os menores de 24 anos têm na maioria (52\% dos entrevistados) não consomem produtos diet. As faixas que mais consomem são as de acima de 45 anos, o que mostra uma possível tendência de idade em relação ao consumo de produtos diet.

GRÁFICO 17: FAIXA ETÁRIA DOS ENTREVISTADOS COM CONSUMO DE PRODUTOS DIET.

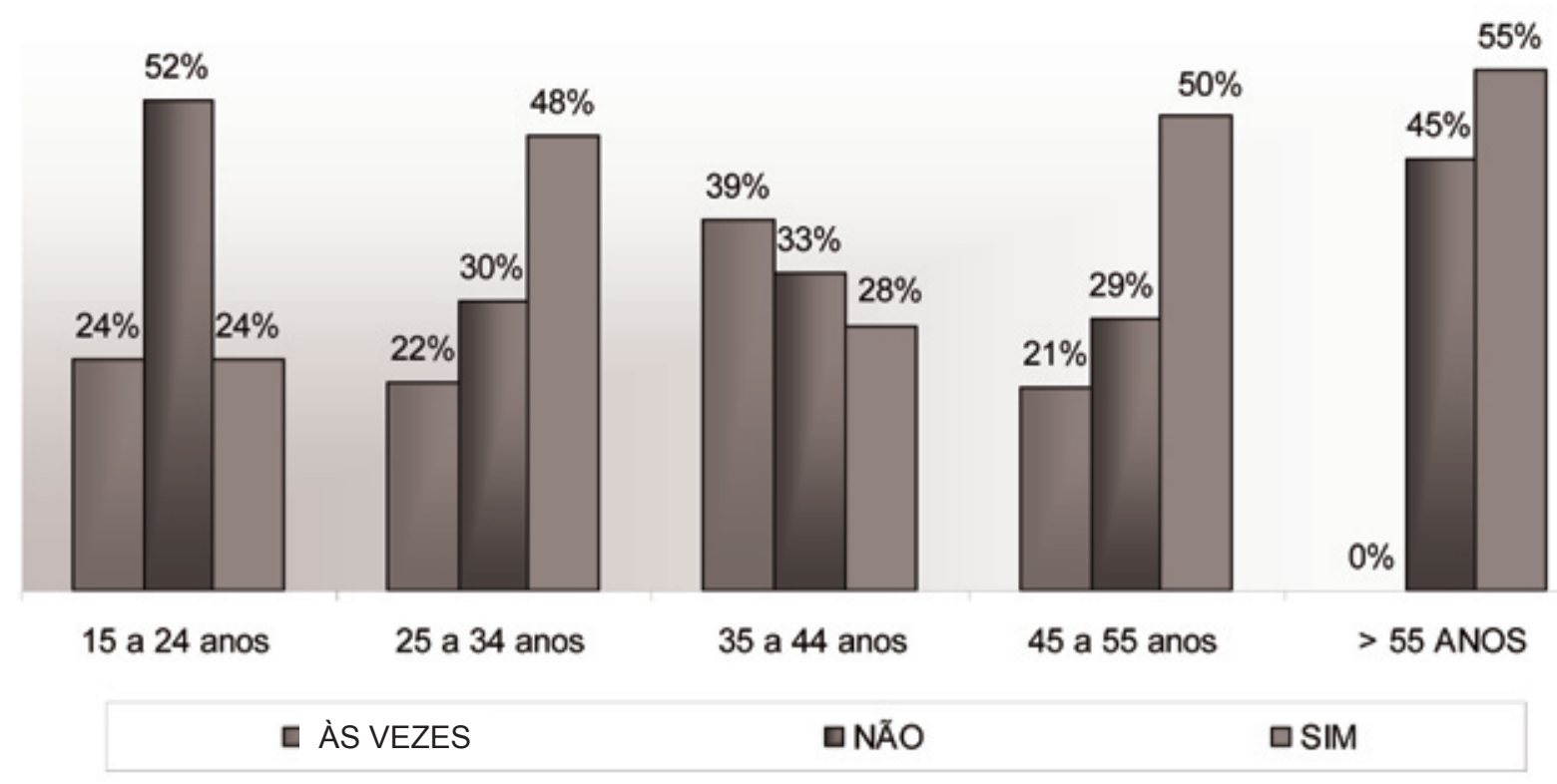


O GRÁFICO 18 cruza os dados de consumo de produtos diet conforme a faixa salarial. Neste caso, temse que quando o salário é bem baixo (menor que um salário mínimo), o consumo de adoçante é baixo, e com freqüência baixa. Na faixa salarial de 4 a 7 salários tem-se mais de $80 \%$ dos entrevistados consumindo produto diet sempre ou as vezes.

GRÁFICO 18: SALÁRIO DOS ENTREVISTADOS EM RELAÇÃO A CONSUMO DOS PRODUTOS DIET.

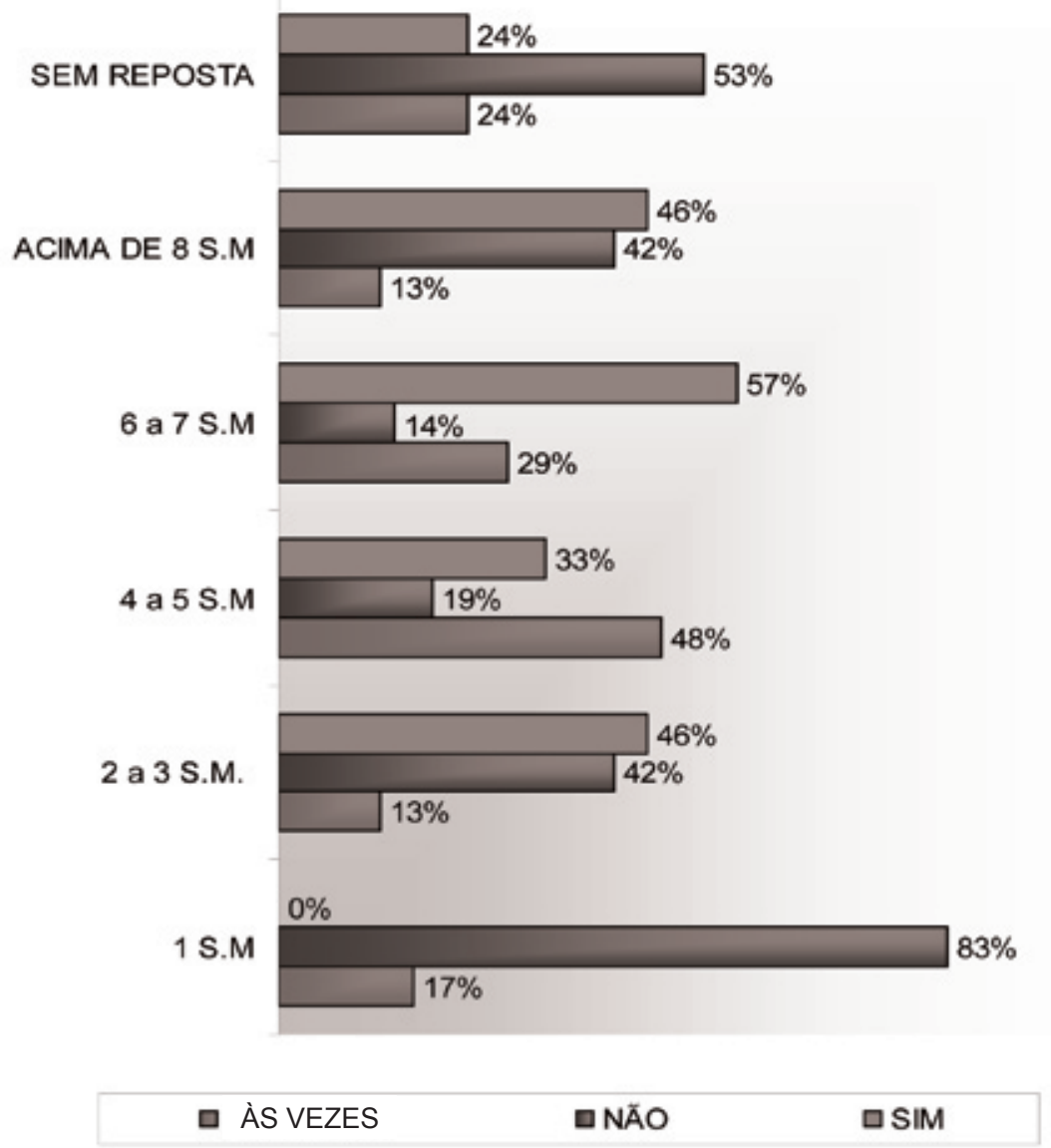

Ao se observar o GRÁFICO 19, que analisa o consumo de produtos diet de acordo com o grau de escolaridade, verifica-se que ele não apresenta nenhuma tendência, pois somando o consumo de "sim" e do "às vezes" temos sempre em torno de $60 \%$ da população das faixas de escolaridade.

GRÁFICO 19: ESCOLARIDADE DOS ENTREVISTADOS.

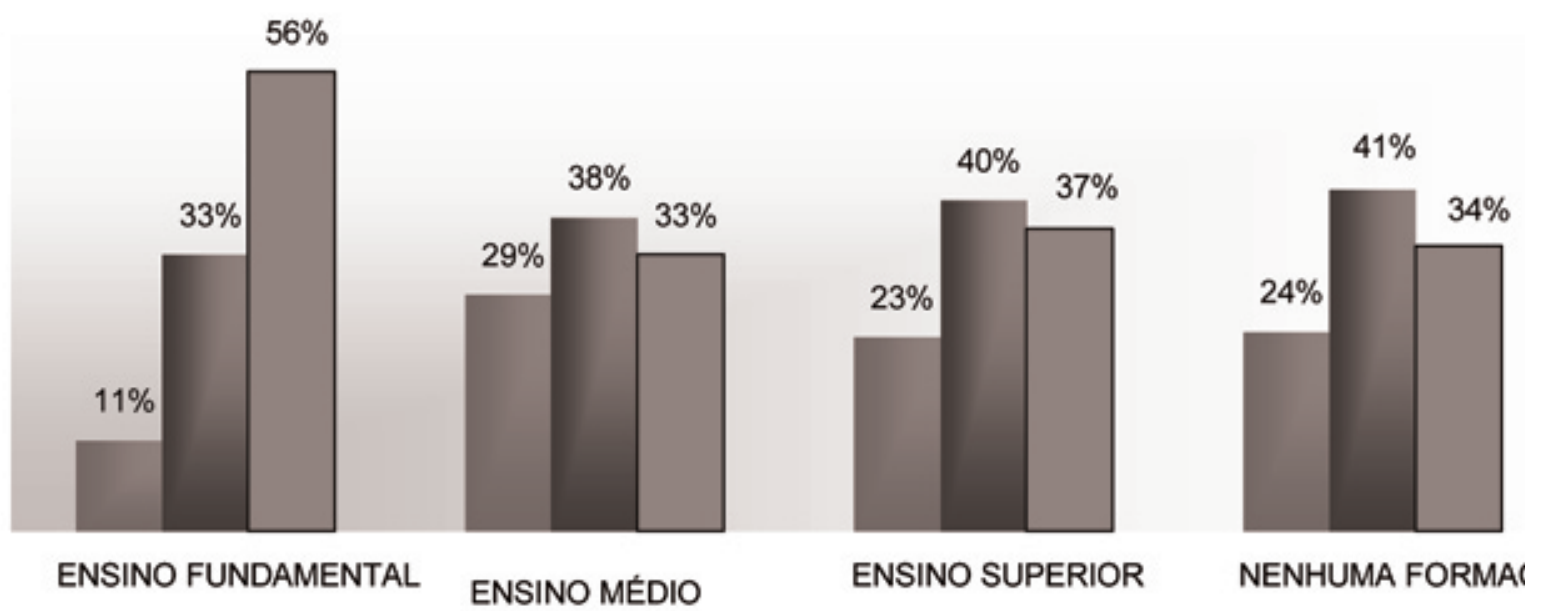

\begin{tabular}{|c|c|c|}
\hline a ÀS VEZES & a NÃO & 口SIM \\
\hline
\end{tabular}


O GRÁFICO 20 mostra que as mulheres apresentam um índice maior de preferência do que os homens por produtos diet. No caso do sexo masculino, aplicando-se o teste qui-quadrado, tem-se com uma confiança de $99 \%$ que não existe preferência por adoçante.

GRÁFICO 20: PREFERÊNCIA POR ADOÇANTES DE ACORDO COM O SEXO DOS ENTREVISTADOS.

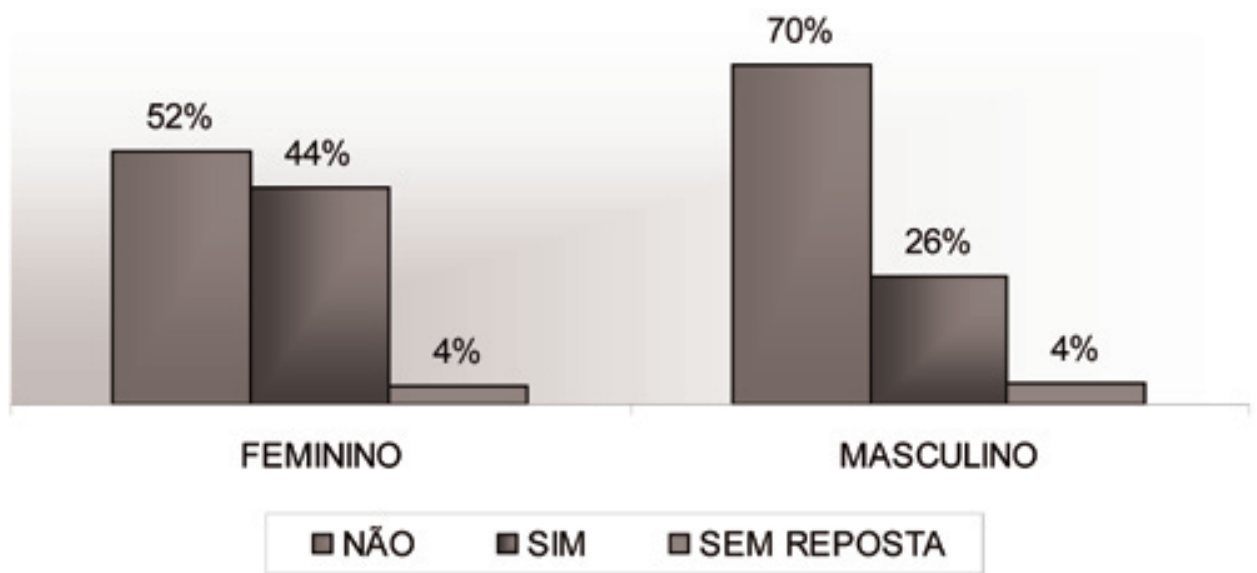

De acordo com o GRÁFICO 21 nota-se que não existe tendência entre fazer academia de ginástica e utilizar produtos diet, o que mostra que a coleta de dados terem mais informações em academias de ginástica não interferiu no objetivo. Ao contrário, dos que freqüentam academia de ginástica às vezes ou caminham às vezes, eles consomem mais produtos diet. No caso dos que não praticam nenhum esporte, eles também se apresentam não consumidores desses produtos (apenas $30 \%$ dos entrevistados).

GRÁFICO 21: CONSUMO DE PRODUTOS DIET EM RELAÇÃO A REALIZAÇÃO DE EXERCíCIOS FísICOS.

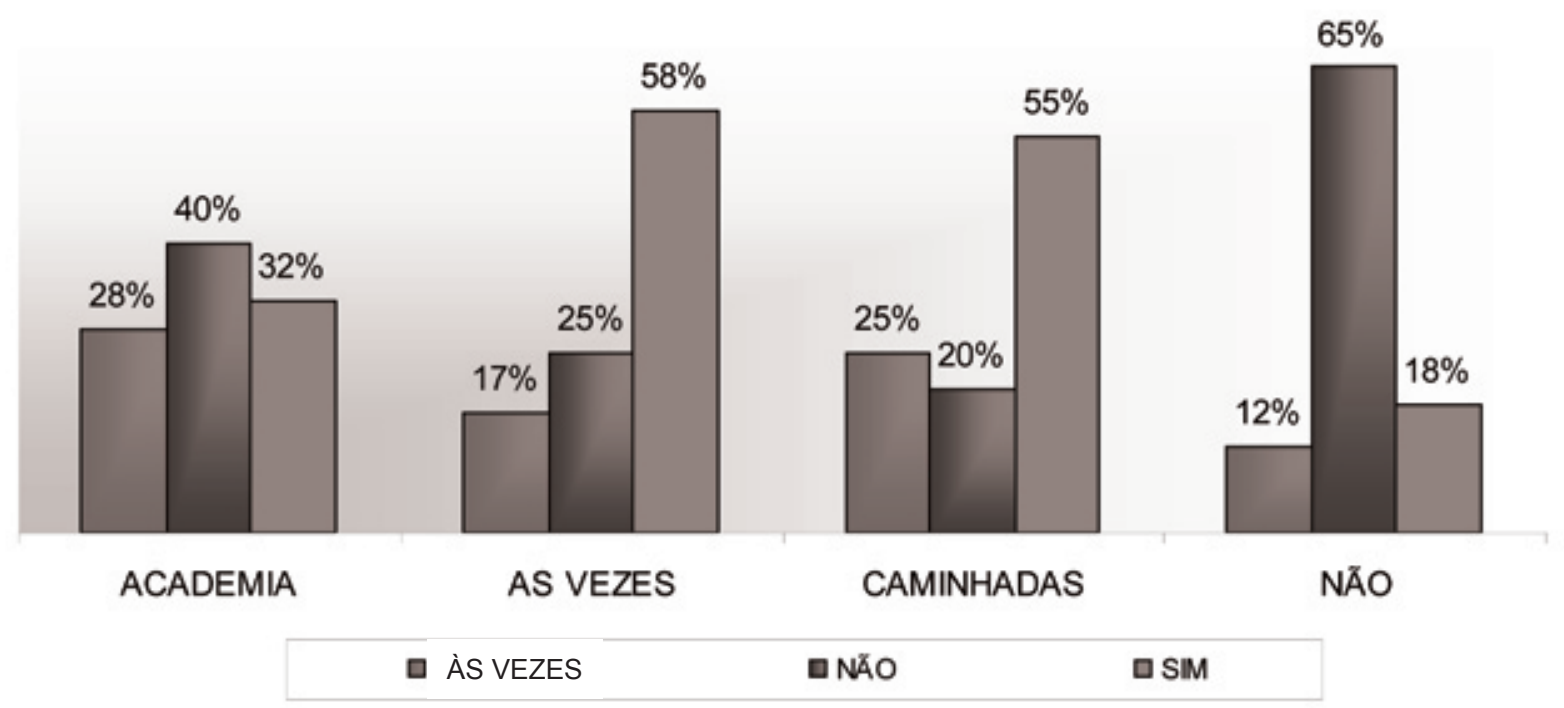

\section{CONCLUSÃO}

A pesquisa mostra que pessoas que possuem patologias como diabetes e obesidade têm tendência a consumir mais produtos diet do que as demais. Um fato interessante é que os indivíduos que freqüentam academia de ginástica, (GRÁFICO 21), na maioria são pessoas que se preocupam mais com a saúde (público de 15 a 24 anos), mostram-se menos consumidoras desses produtos, ou seja, a pesquisa demonstra que não existe tendência 
entre o freqüentar academia de ginástica e o uso de produtos diet. Na verdade, pessoas que caminham às vezes, ou não fazem atividade alguma são os maiores consumidores destes produtos.

Os maiores consumidores são indivíduos acima de 45 anos, ou seja, há uma tendência de idade em relação ao consumo de produtos diet. A maioria dos consumidores dos produtos diet são mulheres, as quais demonstram também ter maior preferência por marca de adoçantes. Observou-se que o grau de escolaridade não interfere no uso destes produtos, ou seja, tanto pessoas com um maior grau de escolaridade do que os com baixo grau, podem consumir tais produtos, porém a renda é um fator considerável, onde faixas salariais mais altas apresentam maior consumo destes produtos.

Observou-se também que a maioria das pessoas consome os produtos diet por conta própria e também que não existe um padrão no objetivo de consumo, ou seja, as pessoas consomem tanto para o bem da saúde como para a estética, mostrando uma desinformação da real funcionalidade dos mesmos. Essa desinformação também é observada quando se analisa o índice de massa corpórea (IMC) dos entrevistados onde $41 \%$ deles apresentam algum tipo de obesidade e apenas $13 \%$ deles sentem-se obesos e citam como uma patologia, ou seja, há uma deficiência dos entrevistados em identificar o que é uma pessoa obesa ou pré-obesa.

Conclui-se, portanto que o consumo de produtos diet não está sendo devidamente orientado. Os consumidores não estão fazendo uso destes produtos com objetivo de melhoria da qualidade da saúde e nem estão procurando orientação médica, nutricionista ou farmacêutica para o uso dos mesmos. As pessoas estão consumindo estes produtos por conta própria e com finalidade de estética e/ou de saúde, correndo sérios riscos de intoxicações ou problemas futuros pelo uso indiscriminado dos mesmos, tanto pela desinformação de suas vantagens e desvantagens, quanto pela desinformação sobre a forma de uso e quantidade recomendada.

É muito importante que os consumidores certifiquem-se melhor do que estão consumindo e qual a real função de um produto diet, para que não haja comprometimentos futuros com a saúde. E, principalmente, sejam realizadas campanhas de orientação pelos órgãos fiscalizadores com Vigilância Sanitária e Ministério da Agricultura no sentido de esclarecer a população sobre o consumo de adoçantes.

\section{REFERÊNCIAS}

AMERINE, Maynard A.; PANGBORN, Rose M.; ROESSLER, Edward B. Principles of sensoy evaluation of food. London: Academic Press, 1965.

CÃNDIDO, Lys Mary Bileski, CAMPOS, Adriane Mulinari. Alimentos para fins especiais: Dietéticos. São Paulo: Varela, 1995.

INMETRO, 2004. Produtos Diet e Light - parte I e II. Disponível em: http://www.inmetro.gov.br/consumidor/produtos/ prodligthdiet1.asp. Acesso em: 07 maio 2006.

TRIOLA, Mario. Introdução à Estatística. 7ª ed. São Paulo: L.T.C.,1999. 


\section{ANEXO 01 - QUESTIONÁRIO DE PESQUISA APLICADO.}

1) Sexo: Feminino ( ) Masculino ( )

2) Faixa etária: ( ) 15 a 24 anos ( ) 25 a 34 anos ( ) 35 a 44 anos ( ) 45 a 55 anos ( ) > 55 ANOS

3) Peso (Kg): Altura (metros):

$\mathrm{IMC}=$ $\mathrm{Kg} / \mathrm{m}^{2}$

4) Renda: ( ) 1 S.M. $\quad$ ( ) 2 a 3 S.M. $\quad$ ( ) 4 a 5 S.M. ( ) 6 a 7 S.M. ( )ACIMA DE 8 S.M.

5)Grau de instrução:

( ) $1^{\circ}$ grau incompleto ( ) $1^{\circ}$ grau completo ( ) $2^{\circ}$ grau incompleto ( ) $2^{\circ}$ grau completo ( ) $3^{\circ}$ grau incompleto ( ) $3^{\circ} \mathrm{grau}$ completo ( ) nenhuma formação

6) Você faz algum exercício físico?

Sim, freqüento academia ( ) Sim, faço caminhadas regularmente ( ) Às vezes Não faço exercício físico ( )

7) Você utiliza produtos diet (sem açúcar) habitualmente?

$\operatorname{Sim}($ ) Não( ) Às vezes ( )

8) Você usa pensando na sua saúde, na estética ou em ambos?

Saúde ( ) Estética ( ) Ambos ( )

9) Usa por conta própria, por recomendação de médico ou recomendação de nutricionista?

( )Uso próprio Recomendação de médico ( ) Recomendação de Nutricionista ( )

10) Você possui alguma patologia? Se sim, qual?

Sim, obesidade ( ) Sim, diabetes ( ) Sim, hipertensão ( ) Não possuo patologia ( )

11) Tem preferência por alguma adoçante? Qual?

$\operatorname{Sim}($ ) Não ( ) Qual: 